\title{
Metrizable Topological Space
}

\author{
Open Mathematics Collaboration*†
}

February 18, 2021

\begin{abstract}
METRIZABLE TOPOLOGICAL SPACE and its underlying definitions are presented in this white paper (knowledge base).
\end{abstract}

keywords: metrizable topological space, metric, topology, knowledge base

The most updated version of this white paper is available at https://osf.io/f8vez/download

\section{Definition}

\section{Metrizable Topological Space}

$\exists d: \mathcal{T}$ is generated from the open balls in $(S, d)$

$(d$ induces $\mathcal{T})$

$S:=$ set

$\mathcal{T}:=$ metric topology on $S$ induced by $d$

$d:=$ metric

$d: S \times S \rightarrow \mathbb{R}$

$[1,2]$

*All authors with their affiliations appear at the end of this white paper.

†Corresponding author: mplobo@uft.edu.br|Open Mathematics Collaboration 


\section{Prerequisites}

2. Metric (distance function)

$$
d: S \times S \rightarrow \mathbb{R}
$$

$S:=$ set
(a) $\forall x, y \in S, \quad(d(x, y)=0) \leftrightarrow(x=y)$
(b) $\forall x, y \in S, \quad d(x, y)=d(y, x)$
(c) $\forall x, y \in S, \quad d(x, z) \leq d(x, y)+d(y, z)$
$[1,2]$

3. Metric Space: $(S, d)$

$S:=$ set

$d:=$ metric

$d: S \times S \rightarrow \mathbb{R}$

$[1,2]$

4. Arbitrary Union

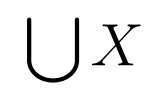

$X:=$ collection of sets

$\bigcup X:=\{y \mid \exists Y \in X, y \in Y\}$

$\cup X$ is the union of the elements of $X$

$[1,2]$ 


\section{Arbitrary Intersection}

\section{$\bigcap X$}

$X:=$ collection of sets

$\cap X:=\{y \mid \forall Y \in X, y \in Y\}$

$\cap X$ is the intersection of the elements of $X$

$[1,2]$

6. Topology on $S$

$S:=$ set

$\mathcal{T}:=$ collection of open subsets of $S$

$X, Y:=$ collection of sets

$\cup X:=$ arbitrary union

$\cap Y:=$ arbitrary intersection

(a) $\varnothing, S \in \mathcal{T}$

(b) $(X \subseteq \mathcal{T}) \rightarrow(\bigcup X \in \mathcal{T})$

[ $\mathcal{T}$ is closed under arbitrary unions]

(c) $(Y \subseteq \mathcal{T}, Y$ finite $) \rightarrow(\cap Y \in \mathcal{T})$

$[\mathcal{T}$ is closed under finite intersections]

$[1,2]$

\section{Topological space}

$S:=$ set

$\mathcal{T}:=$ topology on $S$

$[1,2]$ 
8. Open Ball (center $a$, radius $r$ )

$$
B_{r}(a)=\{x \in S \mid d(a, x)<r\}
$$

$S:=$ set

$d:=$ metric

$(S, d):=$ metric space

$a \in S ; \quad r \in \mathbb{R}^{+}$

$B_{r}(a) \equiv B_{r}(a ; d)$

$[1,2]$

\section{Open Invitation}

Review, add content, and co-author this white paper $[3,4]$.

Join the Open Mathematics Collaboration.

Send your contribution to mplobo@uft.edu.br.

\section{Open Science}

The latex file for this white paper together with other supplementary files are available in [5].

\section{Ethical conduct of research}

This original work was pre-registered under the OSF Preprints [6], please cite it accordingly [7]. This will ensure that researches are conducted with integrity and intellectual honesty at all times and by all means. 


\section{Acknowledgement}

+ Center for Open Science

https://cos.io

+ Open Science Framework

https://osf.io

\section{References}

[1] Warner, Steve. Topology for Beginners. GET 800, 2019.

https://books.google.com/books?id=pNAvxQEACAAJ

[2] Munkres, James R. Topology, 2nd ed. Prentice Hall, 2000.

https://books.google.com/books?id=XjoZAQAAIAAJ

[3] Lobo, Matheus P. "Microarticles." OSF Preprints, 28 Oct. 2019.

https://doi.org/10.31219/osf.io/ejrct

[4] Lobo, Matheus P. "Simple Guidelines for Authors: Open Journal of Mathematics and Physics." OSF Preprints, 15 Nov. 2019.

https://doi.org/10.31219/osf.io/fk836

[5] Lobo, Matheus P. "Open Journal of Mathematics and Physics (OJMP)." OSF, 21 Apr. 2020.

https://doi.org/10.17605/osf .io/6hzyp

https://osf.io/6hzyp/files

[6] COS. Open Science Framework. https://osf .io

[7] Lobo, Matheus P. "Metrizable Topological Space." OSF Preprints, 17 Feb. 2021. https://doi.org/10.31219/osf .io/f8vez 


\section{The Open Mathematics Collaboration}

Matheus Pereira Lobo (lead author, mplobo@uft.edu.br) $)^{1,2}$ https://orcid.org/0000-0003-4554-1372

${ }^{1}$ Federal University of Tocantins (Brazil)

${ }^{2}$ Universidade Aberta (UAb, Portugal) 